\title{
PRODUTIVIDADE DO SORGO GRANÍFERO CV. SACARINO E QUALIDADE DE PRODUTOS FORMULADOS ISOLADAMENTE OU COMBINADOS AO CALDO DE CANA-DE-AÇÚCAR
}

\author{
Cassiara Camelo de SOUZA, José Pires DANTAS , Silvanda de Melo SILVA ; \\ Vênia Camelo de SOUZA", Fernandes Antonio de ALMEIDA“, Luiz Eloi da SILVA
}

\begin{abstract}
RESUMO
Este trabalho teve como objetivo avaliar o comportamento produtivo do Sorgo granifero (Sorghum bicolor L) variedade IPA- 467, mediante condições de irrigação e adubação, bem como, a caracterização físico-química da farinha de diferentes tipos de grãos e de rapadura obtida a partir de combinações de caldo de sorgo (CS) x caldo de cana (CC). O experimento resultou em uma produção de biomassa, sementes, colmo, caldo, melaço fino e melaço grosso, respectivamente, de: $64 \mathrm{t} / \mathrm{ha} ; 3,5 \mathrm{t} / \mathrm{ha} ; 46 \mathrm{t} / \mathrm{ha} ; 700 \mathrm{~L} / \mathrm{t}$ de colmo; $140 \mathrm{~L} / \mathrm{t}$ de colmo e $90 \mathrm{~L} / \mathrm{t}$ de colmo. A farinha obtida a partir de grãos de sorgo apresenta teor de açúcares totais inferior aos da farinha de trigo. As rapaduras em que o caldo de sorgo foi adicionado nas proporções de 10 e $20 \%$, em associação com o caldo-decana, obtiveram maior nível de aceitação, quando comparada à rapadura obtida a partir de $100 \%$ de caldo de cana-de-açúcar. A aceitação de rapaduras formuladas a partir 30\% de caldo sorgo e $70 \%$ de caldo de cana não diferiu de rapaduras obtidas de $100 \%$ de caldo de cana.

Palavras-chave: qualidade; Sorghum bicolor; rapadura; melaço; caracterização físico-química.
\end{abstract}

\section{SUMMARY}

YIELD OF SORGHUM BICOLOR CV. SACARINO AND QUALITY OF PRODUCTS FORMULATED ISOLATED OR COMBINED WITH SUGAR CANE JUICE. The objective of this work was to evaluate the yield of Sorghum bicolor var. IPA-467, under irrigation and fertilizer conditions and the physical-chemical characterization of grain flours and 'rapadura' obtained from combinations of sorghum stem juice (SJ) x sugar cane juice (CJ). The experiment resulted im biomass production, seeds, stem, juice, thick sugarcane syrup, respectively of $64 \mathrm{t} / \mathrm{ha} ; 3,5 \mathrm{t} / \mathrm{ha}, 46 \mathrm{t} / \mathrm{ha}, 700 \mathrm{~L} / \mathrm{t}$ of stem, $140 \mathrm{~L} / \mathrm{t}$ of stem and $90 \mathrm{~L} / \mathrm{t}$ of stem. The total sugars of sorghum grain flour were lower than results reported for wheat flour. 'Rapaduras' in which sorghum juice was used at 10 and 20 , in combination of sugar cane juice, had higher acceptance scores, as compared with 'rapadura' obtaned from $100 \%$ sugar cane juice. The acceptance of 'rapaduras' formulated from 30\% SJ x 70\% CJ did not differ from those obtained from $100 \%$ CJ.

Keywords: quality; Sorghum bicolor; 'rapadura'; 'melaço'; physical-chemical characterization.

\section{1-INTRODUÇÃO}

Entre as culturas de grande potencial energético as de maior destaque são a cana-de-açúcar, a beterraba açucareira e sorgo sacarino. Dessas culturas, a canade-açúcar se desenvolve bem no trópico úmido, enquanto a beterraba açucareira se desenvolve em clima temperado [9]. O sorgo sacarino se assemelha à cana-deaçúcar, uma vez que o armazenamento do açúcar ocorre no colmo, além de fornecer bagaço para a indústria [13]. Entretanto, ele difere de maneira acentuada da cana-deaçúcar pelo fato de ser cultivado a partir de sementes e apresentar um ciclo vegetativo bem mais curto, de 120 a 130 dias. Adicionalmente, o sorgo sacarino produz grãos, que podem ser utilizados na alimentação humana [15], além de reconhecidamente, uma opção economicamente viável na alimentação animal [13].

Recebido para publicação em 21/07/2004. Aceito para publicação em 11/08/2005(001380).

Programa DTI-F.

Universidade Estadual da Paraíba-UEPB.

DCFS/CCA/Universidade Federal da Paraíba-UFPB. Email:silvasil@cca.ufpb.br

DCBS/CFT/UFPB. CEP: 58397-000,Bananeiras-PB.

'Programa Pós-Graduação em Produção Vegetal

CEFET-BA

A quem a correspondência deve ser enviada.
O sorgo granifero cv. sacarino [Sorghum bicolor (L.) Moench] é uma das culturas líder do mundo agrícola, embora ainda seja pouco conhecido na América e na Europa. É o quinto cereal mais plantado no mundo, vindo logo depois do trigo, arroz, milho e cevada. No Brasil, o sorgo tem despontado como excelente alternativa para as diversas regiões brasileiras, com produção anual de 242.745 toneladas em 1995. No Nordeste brasileiro, a aplicação do sorgo na alimentação humana tem grandes potencialidades de uso e poderá constituir considerável economia de divisas, com a diminuição da importação do trigo [14].

Este cereal possui tolerância a períodos de estiagem durante seu ciclo vital e produz colheitas de grãos e massa verde, economicamente compensadoras, em condições de pluviosidade baixa ou instável, até em solos de má qualidade [4]. Assim, o sorgo é cultivado principalmente em zonas áridas e semi-áridas, tornando-se um alimento básico, visto que apresenta: elevado potencial de produção, reconhecida qualificação como fonte de energia para arroçoamento animal, grande versatilidade (silagem, feno e pastejo direto) e potencial de adaptação a regiões mais secas [12], com boa produtividade de grãos e altos teores de açúcares no caldo do colmo [15], além de elevada saturação lumínica e maior tolerância ao déficit hídrico e à salinidade, caracterizando-se com as especificidades do bioma caatinga [10].

O sorgo é um cereal cultivado na maior parte das re- 
giões tropicais e subtropicais do mundo, constituindo a maior fonte de alimento e de rações da África, Oriente e Oriente Médio, especialmente na Nigéria, Etiópia e Índia [11]. Em muitas regiões da África, o sorgo constitui um dos alimentos básicos da dieta humana, suprindo cerca de 70 \% da ingestão calórica diária. Nos Estados Unidos, a farinha de sorgo é adicionada à farinha de trigo, para fabricação de muitos produtos alimentícios como pães, biscoitos, mingaus, além do melado obtido do caldo [13].

A rapadura obtida de cana-de-çúcar é um produto amplamente utilizado pela população nordestina [3], sobretudo pela população de baixa renda. Diante de tanta versatilidade, admite-se que o sorgo possa ser também introduzido na alimentação humana [13], inclusive na formulação de produtos como o melaço e a rapadura, ampliando a sua utilização como alimento, possibilitando a atenuação da situação alimentar da faixa de renda mais pobre da região Nordeste. Esta tarefa que deve ser levada a efeito com bastante rapidez, tendo em vista os graves prejuizos que o problema de subnutrição poder acarretar ao esforço de desenvolvimento regional, ora em execução, a exemplo do Programa do Governo Federal, o Fome Zero. Em razão disso, tem-se que pensar em minorar o déficit através produtos de origem vegetal, que são muito mais acessiveis e, portanto, estão ao alcance dos grupos mais afetados. É, portanto, dentro deste contexto que se pretende situar o sorgo granífero, cereal que pode dar uma grande contribuição para solução do problema de segurança alimentar e qualidade de vida do homem do Nordeste.

Baseado no exposto, o presente trabalho teve por objetivo avaliar a produtividade dessa cultura mediante condições de irrigação e adubação, bem como proceder a caracterização físico-química da farinha de grãos, do melaço do caldo do colmo e de rapaduras formuladas a partir do caldo do sorgo granífero cv. Sacarino associado ao caldo de cana-de-açúcar.

\section{2 - MATERIAL E MÉTODOS}

\section{1 - Condições experimentais}

Foram implantadas duas áreas de produção em condições de campo para realização deste trabalho. A primeira área de produção, destinada à obtenção do colmo para obtenção de caldo e dos subprodutos deste, foi instalado na Escola Agrícola Assis Chateaubriand UEPB, no município de Lagoa Seca, estado da Paraíba. Plantouse uma área de $600 \mathrm{~m}^{2}$ com sorgo granífero cv. Sacarino, variedade IPA- 467, com espaçamento entre sulcos de $90 \mathrm{~cm}$ colocando-se 30 sementes por metro linear de sulco. No plantio usou-se adubação mineral composta de $20 \mathrm{~kg}$ de $\mathrm{N}, 80 \mathrm{~kg}$ de $\mathrm{P}_{2} \mathrm{O}_{s}$ e $60 \mathrm{~kg}$ de $\mathrm{K}_{2} \mathrm{O}$ nas formas respectivas de sulfato de amônio, superfosfato triplo e cloreto de potássio. Transcorridos 30 dias do plantio procedeuse cobertura com $40 \mathrm{~kg}$ de $\mathrm{N}$ na forma de uréia. Durante o transcorrer do desenvolvimento das plantas procedeu-se capinas.
A segunda área de produção, utilizada com o objetivo de se determinar parâmetros de produção em condições de irrigação, foi instalada às margens do açude Marechal Dutra, município de Acari, estado do Rio Grande do Norte, microrregião do Seridó, devido à disponibilidade de água para irrigação. Nesta área foi empregada a mesma adubação empregada na primeira área experimental, acrescida de 10t/ha de esterco bovino como adubação orgânica. O plantio do sorgo realizou-se em pequenos sulcos abertos lateralmente nos taludes dos sulcos de irrigação, utilizando-se o espaçamento entre os pequenos sulcos de plantio de $60 \mathrm{~cm}$ e colocando-se o número de aproximadamente 30 sementes por metro linear de sulco. Utilizou-se o sistema de irrigação em sulco com turno de rega até os 4 dias do plantio de 4 dias e a partir do $4^{\circ}$ dia até 10 dias da colheita de 7 dias. Procedeu-se a colheita após 3 meses do plantio. As panículas do sorgo e os grãos foram destacados dos colmos e colocados a secar ao sol. Os grãos, após atingirem cerca de $13 \%$ de umidade, foram destacados da panícula e armazenados para posterior preparação da farinha e procedimento da caracterização físico-química.

\section{2 - Preparação de produtos e avaliações}

\subsection{1 - Farinha de grãos}

Tomando-se como base as características fenotipicas dos grãos, por haver segregação no sorgo, separou-se três tipos de grãos, denominados de tipos A, B e C, considerando-se cada tipo como um tratamento. Estes foram descortiçados, aquecidos em estufa a $105^{\circ} \mathrm{C}$ por 1 hora, triturados em moinho para obtenção de farinha e avaliados, conforme metodologia descrita em INSTITUTO ADOLFO LUTZ [8], quanto às seguintes características: umidade, cinzas, $\mathrm{pH}$, acidez, amido e açúcares totais, em cinco repetições, exceto para as duas últimas características, onde foram tomadas três repetições.

\subsection{2 - Preparação de produtos a partir do caldo do colmo}

A caracterização dos produtos obtidos a partir do caldo extraído do colmo do sorgo granifero, obedeceu a seguinte metodologia: obteve-se o caldo a partir da moagem do colmo no engenho de cana-de-açúcar Vaca Brava, localizado no município de Areia-PB, procedendose a determinação do teor de sólidos solúveis totais, com refratômetro manual (ATAGO), sendo os resultados expressos em porcentagem. Após a extração o caldo do colmo do sorgo foi filtrado em peneira plástica de malha fina e, em seguida, submetido ao cozimento até a obtenção do melaço.

Para avaliação de misturas entre o caldo do colmo do sorgo $\mathrm{x}$ caldo do colmo da cana na composição de produtos, tomou-se cana-de-açúcar do canavial do engenho para a obtenção do caldo, determinando-se também o seu teor de sólidos solúveis, procedendo-se em seguida o cozimento até a obtenção do melaço.

Para preparação da rapadura, após a obtenção dos 
melaços de caldo do sorgo (CS) e do caldo de cana (CC), foram formuladas misturas que consistiram nos seguintes tratamentos: 1) $0 \% \mathrm{~V} / \mathrm{V} \mathrm{CS} \times 100 \% \mathrm{CC}$; 2) $10 \% \mathrm{~V} / \mathrm{V}$ CS x $90 \% \mathrm{CC}$; 3) $20 \% \mathrm{~V} / \mathrm{V} \mathrm{CS} \times 80 \% \mathrm{CC}$; 4) $30 \% \mathrm{~V} / \mathrm{V} \mathrm{CS}$ x 70\% CC; 5) 40\% V/V CS x 60\% CC; 6) $50 \%$ V/V CS x $50 \%$ CC e 7) $100 \%$ V/V CS x 0\% CC. Após a formulação, as misturas obtidas de cada tratamento foram conduzidas ao engenho para a obtenção de rapaduras.

As rapaduras foram avaliadas quanto aos teores de: umidade, cinzas, acidez, $\mathrm{pH}$, glicose e sacarose, segundo o INSTITUTO ADOLFO LUTZ [8], utilizando-se três repetições para as determinações de glicose e sacarose e cinco, para as demais análises.

Para a avaliação da aceitação geral das rapaduras obtidas nas formulações 10\% CS x 90\% CC, $20 \%$ CS x $80 \%$ CC, $30 \%$ CS x $70 \%$ CC e $100 \%$ CC, foram utilizados 43 provadores não-treinados. A ficha empregada foi de escala hedônica não-estruturada de 10 pontos, ancorada nos seus extremos com as impressões "desgostei muitíssimo" (escore = 1) e "gostei muitíssimo" (escore = 10). As amostras foram servidas aleatoriamente em cabines individuais.

As rapaduras obtidas a partir das proporções de $40 \%$ CS x $60 \%$ CC e $50 \%$ CS x $50 \%$ CC não foram avaliadas, devido a não se apresentarem bem cristalizadas e de boa textura.

\section{3 - Análise estatística}

A análise estatística dos dados foi realizada usando o programa da UNESP- FCAV- Campus de Jaboticabal. Polo Computacional/ Departamento de Ciências Exatas. Estat-Sistema para análises estatísticas (V.2.0). Utilizou-se o teste de Tukey ao nivel de $(\mathrm{p} \leq 0,05)$ para comparação de médias dos parâmetros obtidos em função dos tratamentos.

\section{3 - RESULTADOS E DISCUSSÃO}

Os resultados referentes aos componentes de produção do sorgo granífero cv. Sacarino em condições de irrigação e adubação orgânica e mineral encontram-se na Tabela 1. A produtividade de massa verde total deste trabalho foi bastante alta, obtendo-se o valor de $64 \mathrm{t} / \mathrm{ha}$, acima do rendimento agricola que fica entre 40 a 50t/ha e dos resultados encontrados por CAMACHO et al. [4], que trabalhando com 10 variedades de sorgo granifero cv. sacarino com a finalidade de testar o comportamento agronômico das cultivares, obteveram um rendimento médio de massa verde total de 35,97t/ha.

A produção de folhas e de colmo de sorgo deste experimento foi de respectivamente 18t/ha e 46t/ha, sendo superiores aos relatados por MARCHEZAN [11], que realizando experimento do ensaio nacional do sorgo sacarino em Santa Maria-RS, com o objetivo de identificar genótipos com alto potencial produtivo em condições de campo, testaram 10 variedades de sorgo, obtendo um rendimento médio de 5,02t/ha de folhas e 31,50t/ha de colmo.

Quanto aos dados de produtividade das sementes obteve-se rendimento de $3,5 \mathrm{t} /$ ha sendo similar aos reportados por CHIELLE [6], que estudando duas variedades de sorgo em condições de campo e irrigação, obtiveram produção média de sementes de $3,86 \mathrm{t} / \mathrm{ha}$, concluindo que esta produção foi diretamente proporcional à população de plantas. A alta produtividade expressa nas características morfológicas de biomassa, matéria seca de bagaço, produção de sementes, colmo e folhas constatam o excelente crescimento vegetativo desta planta e sua resposta favorável à adubação orgânica e mineral aplicadas. A produção de caldo assim como melaços fino e grosso também demonstram a capacidade produtiva desta graminea, chegando-se aos valores de 700, 140 e 90L/tonelada de colmo, respectivamente.

No transcorrer do cultivo não se constatou nenhuma incidência de praga e/ou doença, o que vem a comprovar a alta contribuição que o sistema de produção dessa cultura poderá oferecer ao meio ambiente.

Com relação ao teor de sólidos solúveis do caldo do colmo do sorgo, evidenciou-se que o seu valor atingiu a variação de 14-18,5\%, constatando-se a presença de açúcares dissolvidos que, quantitativamente, equivalem aos determinados no caldo da cana-de-açúcar que atingiu os valores de $16-19 \%$.

Nos engenhos de cana do Nordeste, a rapadura é um produto altamente produzido e amplamente consumido no mercado interno, consistindo num importante fonte de emprego e renda, além de carboidrato, para a população [3]. Os resultados dos parâmetros químicos determinados nos produtos obtidos a partir dos caldos da cana (CC), do sorgo (CS) e de formulações de caldo de cana (CC) x caldo de sorgo (CS) encontram-se na Tabela 2.

O teor de umidade das rapaduras obtidas variou de 6,20 a $8,53 \%$, representando respectivamente os tratamentos $100 \%$ CC e $20 \%$ CS x $70 \%$ CC que diferiram sig-

TABELA 1 - Componentes de produção do sorgo granífero cv. Sacarino em condições de irrigação e adubação orgânica e mineral (Acari RN, 2003)

Componentes de Produção

\begin{tabular}{ccccccc}
\hline $\begin{array}{c}\text { Biomassa } \\
\text { (t/ha) }\end{array}$ & $\begin{array}{c}\text { Bagaço } \\
{ }^{*} \text { MS (\%) }\end{array}$ & $\begin{array}{c}\text { Grãos } \\
\text { (t/ha) }\end{array}$ & $\begin{array}{c}\text { Colmo } \\
\text { (t/ha) }\end{array}$ & $\begin{array}{c}\text { Folhas } \\
\text { (t/ha) }\end{array}$ & $\begin{array}{c}\text { Melaço Fino } \\
(\mathrm{L} / \mathrm{t} \text { de colmo })\end{array}$ & $\begin{array}{c}\text { Melaço Grosso } \\
(\mathrm{L} / \mathrm{t} \text { de colmo })\end{array}$ \\
\hline "MS (Matéria Seca) & 48,3 & 3,3 & 46 & 18 & 700 & 90 \\
\hline
\end{tabular}


TABELA 2 - Características físico-químicas de produtos do caldo de sorgo (CS), do caldo de cana-de-açúcar (CC) e de combinações de caldo de sorgo e caldo de cana-de-açúcar (CS x CC)

\begin{tabular}{ccccccc}
\hline Tratamento & Produto & $\begin{array}{c}\text { Umidade } \\
(\%)\end{array}$ & $\begin{array}{c}\text { Cinzas } \\
(\%)\end{array}$ & $\begin{array}{c}\text { Acidez } \\
(\mathrm{mL} \mathrm{NaOH} \mathrm{N})\end{array}$ & $\mathrm{pH}$ & $\begin{array}{c}\text { Açúcares } \\
\text { Totais } \\
(\%)\end{array}$ \\
\hline $100 \% \mathrm{CS}$ & Melaço & $25,91 \mathrm{a}^{\star}$ & $6,26 \mathrm{a}$ & $14,46 \mathrm{a}$ & $5,42 \mathrm{e}$ & $49,12 \mathrm{e}$ \\
$100 \% \mathrm{CC}$ & Rapadura & $6,20 \mathrm{~d}$ & $1,60 \mathrm{~d}$ & $4,42 \mathrm{~b}$ & $6,56 \mathrm{c}$ & $82,68 \mathrm{a}$ \\
$10 \% \mathrm{CS} \mathrm{x}$ & Rapadura & $7,46 \mathrm{c}$ & $1,76 \mathrm{bc}$ & $2,74 \mathrm{c}$ & $5,90 \mathrm{~d}$ & $79,15 \mathrm{~b}$ \\
$90 \% \mathrm{CC}$ & & & & & & \\
$20 \% \mathrm{CS} \times$ & Rapadura & $8,53 \mathrm{~b}$ & $1,95 \mathrm{bc}$ & $2,64 \mathrm{c}$ & $6,74 \mathrm{~b}$ & $76,61 \mathrm{c}$ \\
$\begin{array}{c}80 \% \mathrm{CC} \\
30 \% \mathrm{CS} \times\end{array}$ & Rapadura & $8,31 \mathrm{bc}$ & $2,39 \mathrm{~b}$ & $2,88 \mathrm{c}$ & $6,86 \mathrm{a}$ & $77,13 \mathrm{bc}$ \\
$70 \% \mathrm{CC}$ & & & & & & \\
$\begin{array}{c}\text { D.M.S } \\
\text { (p?0,05) }\end{array}$ & 0,9971 & 0,6551 & 0,7376 & 0,0887 & 1,4144 \\
\hline
\end{tabular}

nificativamente entre si e das demais amostras. Observou-se aumento no teor de umidade dos produtos classificados como rapadura à medida que aumentava a proporção de caldo de sorgo à constituição do produto.

Nos dados encontrados na determinação de cinzas, observou-se diferenças significativas entre rapaduras constituídas de $100 \%$ CC e as formuladas a partir de misturas de CC x CS. Entre os produtos classificados como rapadura, observou-se que o teor de cinzas aumentava à medida que a concentração do sorgo no produto tornavase mais elevada. Convém ressaltar, no entanto, que o maior valor para o teor de cinzas foi detectado no melaço $(6,26 \%)$.

Com relação à acidez, evidenciou-se diferenças significativas entre o produto classificado como melaço (14, $46 \mathrm{~mL}$ ) e a rapadura produzida exclusivamente com cana-de-açúcar $(4,42 \mathrm{~mL})$, e entre estes e os tratamentos que possuem relações variáveis sorgo/cana, onde os valores oscilaram de 2,64 a $2,88 \mathrm{~mL}$.

Quanto aos dados encontrados para $\mathrm{pH}$, constatouse diferenças significativas entre todos os tratamentos $(\mathrm{p} \leq 0,05)$, evidenciando-se que, o melaço obtido exclusivamente a partir do caldo de sorgo, pode ser considerado de caráter mais ácido $(5,42)$, porém, à medida que a concentração de sorgo aumentava, na rapadura fabricada a partir de combinações sorgo x cana, observava-se a elevação do $\mathrm{pH}$.

Constatou-se que o melaço obtido a partir de $100 \%$ do caldo do colmo de sorgo apresentou teor de açúcares totais de $49,12 \%$. Para produtos derivados da cana-deaçúcar, a ANVISA [1] determina um teor mínimo de 50\% $(\mathrm{p} / \mathrm{p})$ para glicídios totais, para o produto designado como 'melaço de cana', valor aproximado ao obtido neste experimento para 'melaço de sorgo'.

Em relação aos teores de açucares totais (AT) das rapaduras, observou-se diferenças significativas a $5 \%$ de probabilidade, entre os produtos fabricados com 10, $20 \mathrm{e}$ $30 \%$ de sorgo com relação à rapadura obtida a partir de $100 \%$ caldo de cana-de-açúcar. Rapadura obtida exclusivamente a partir de cana-de-açúcar apresentou o maior teor de AT $(82,68 \%)$ e observou-se um decréscimo dos açúcares totais em rapaduras à medida que a concentração do sorgo aumentava. Para rapadura de canade-açúcar, a ANVISA [1] determina um teor mínimo de $80 \%(\mathrm{p} / \mathrm{p}$ ) para glicídios totais, para o produto designado como 'rapadura. Os valores inferiores a $80 \%$ nos glicídios totais observados para rapaduras formuladas em associação com o caldo do colmo do sorgo podem ser decorrentes de dificuldades do operador de tacho atingir o 'ponto' do produto final, visto que esta tratava-se de uma formulação não utilizada no engenho de cana.

A Tabela 3 apresenta as notas médias obtidas pela análise sensorial das rapaduras obtidas a partir de quatro formulações. Pode-se observar que a rapadura formulada a partir de $90 \%$ CC x $10 \%$ CS foi a preferida pelos avaliadores, obtendo média de 8,63. A formulação de rapadura $70 \%$ CC x 30 \% CS obteve média de aceitação similar à obtidas por rapadura formulada a partir de $100 \%$ de caldo de cana-de-açúcar. Rapaduras formuladas a partir de caldo de sorgo x caldo de cana, segundo os avaliadores, apresentavam-se mais macios, de melhor textura e aroma mais agradável, quando comparado à rapaduras formuladas exclusivamente a partir de caldo de cana. As rapaduras obtidas apresentavam-se como de boa consistência e bem cristalizada e de excelente palatabilidade.

TABELA 3 - Médias da aceitação geral de rapaduras obtidas a partir de caldo de cana-de-açúcar (CC) e de formulações de caldo de sorgo (CS) e caldo de cana-de- açúcar (CS x CC), Areia$\mathrm{PB}, 2003$

\begin{tabular}{|c|c|c|c|c|}
\hline & \multicolumn{4}{|c|}{ Formulação } \\
\hline & $100 \% \mathrm{CC}$ & $90 \%$ CC $\times 10 \%$ CS & $80 \%$ CC $\times 20 \%$ CS & $70 \%$ CC $\times 30 \%$ CS \\
\hline $\begin{array}{l}\text { Aceitaçăo } \\
\text { Geral }\end{array}$ & $7,49 \mathrm{c}^{*}$ & $8,63 \mathrm{a}$ & $7,68 \mathrm{~b}$ & $7,56 \mathrm{c}$ \\
\hline
\end{tabular}

As características físico-químicas de farinhas obtidas a partir de três tipos de grãos do sorgo granífero cv. sacarino encontram-se na Tabela 4.

Segundo RASPER [14] a qualidade de grãos e farinhas é determinada por uma variedade de características, as quais podem ser divididas em fisicas, químicas, enzimáticas e reológicas. Analisando-se as características fisico-quimicas, observou-se que as farinhas dos grãos de sorgo apresentaram teor de umidade de 7,48 a $8,81 \%$, onde os tipos B e C diferiram significativamente entre si. Para a farinha de trigo, o grau máximo de umidade permitido pela legislação brasileira [2] é de 15\%, estando as farinhas dos três tipos de sorgo avaliadas, portanto, abaixo deste limite. A disponibilidade de umidade é importante por ser um dos principais fatores de aceleração das reações enzimáticas.

O teor de cinzas da farinha de trigo é usado na legislação brasileira para classificar a farinha de uso doméstico entre especial e comum. Para esta ser classificada como especial, o teor de cinzas deve ser inferior a 0,65\% [2]. Os valores encontrados neste trabalho para farinha de 
TABELA 4 - Características físico-químicas de farinhas obtidas a partir de três tipos de grãos do sorgo granífero cv. Sacarino (Acari RN, 2003)

\begin{tabular}{|c|c|c|c|c|c|c|}
\hline $\begin{array}{c}\text { Tipo de } \\
\text { Grão }\end{array}$ & $\begin{array}{c}\text { Umidade } \\
(\%)\end{array}$ & $\begin{array}{c}\text { Cinzas } \\
(\%)\end{array}$ & $\begin{array}{c}\text { Acidez } \\
(\mathrm{mL} \mathrm{NaOH} \mathrm{N})\end{array}$ & $\mathrm{pH}$ & $\begin{array}{c}\text { Amido } \\
(\%)\end{array}$ & $\begin{array}{c}\text { Açúcares } \\
\text { Totais } \\
(\%)\end{array}$ \\
\hline Tipo A & $7,73 \mathrm{ab}^{*}$ & $1,80 \mathrm{~b}$ & 2,95 a & $6,42 \mathrm{a}$ & $51,15 b$ & $56,84 \mathrm{c}$ \\
\hline Tipo B & $7,48 \mathrm{~b}$ & $1,98 \mathrm{a}$ & 2,76 a & $6,28 \mathrm{a}$ & $52,80 \mathrm{~b}$ & 58,97 b \\
\hline Tipo C & 8,81 a & $1,87 \mathrm{~b}$ & $3,24 \mathrm{a}$ & $6,30 \mathrm{a}$ & 61,96 a & 68,85 a \\
\hline $\begin{array}{c}\text { D.M.S } \\
(\mathrm{p} \leq 0,05)\end{array}$ & 1,2221 & 0,0980 & 0,5002 & 0,1508 & 1,8818 & 2,0939 \\
\hline
\end{tabular}

grãos de sorgo oscilaram entre 1,80 a 1,98\%, sendo o teor de cinzas da farinha de grãos do tipo B superior $(p \leq 0,05)$ aos dos demais tipos e, possivelmente, detentora de maior concentração de minerais. Comparativamente a farinha de trigo, estes valores foram superiores aos reportados por GUTKOSKI \& NETO [7] que caracterizaram cinco tipos de farinha e encontraram o valor médio de 0,89\%.

Nos resultados obtidos para o teor de acidez, não foram observadas diferenças significativas entre as farinhas dos três tipos de grãos, sendo a farinha obtida a partir de grãos do tipo $\mathrm{C}$ a que apresentou maior acidez $(3,24 \mathrm{~mL} \mathrm{NaOH} 0,1 \mathrm{~N})$ e a do tipo B, a menor $(2,76 \mathrm{~mL}$ $\mathrm{NaOH} 0,1 \mathrm{~N})$. Os dados referentes ao $\mathrm{pH}$, não apresentaram diferença significativa entre as farinhas dos três tipos de grãos de sorgo, destacando-se a farinha do tipo A que foi detentora de $\mathrm{pH}$ mais próximo da neutralidade $(6,42 \mathrm{~mL} \mathrm{NaOH} 0,1 \mathrm{~N})$.

Os dados referentes ao teor de amido e açúcares totais revelaram que grãos dos tipos A e B não apresentam diferenças significativas entre si, porém diferem consideravelmente do tipo $\mathrm{C}$ que se mostrou com maior concentração de amido $(61,96 \%)$ e açúcares totais $(68,85 \%)$ em seus grãos.

CARVALHO \& NAKAGAWA [5] relatam que o teor de amido do sorgo corresponde a $65 \%$ a $75 \%$ do grão inteiro, representando a maior parte do seu conteúdo de carboidrato. Neste trabalho, os valores obtidos para o teor de amido dos grãos avaliados identificaram o seu alto poder de concentração desse carboidrato, visto que os grãos foram descortiçados, e esta prática, promove a diminuição do teor amiláceo, já que na bráctea também se encontram consideráveis niveis de amido. O conteúdo de açúcares totais da farinha de grãos de sorgo variou de 56,84 a $68,85 \%$ e estes valores mostraram-se inferiores aos reportados para a farinha de trigo por TREDUS et al. [16], que detectaram $75,08 \%$ de açúcares totais. Porém, farinha obtida a partir de grãos de sorgo do tipo C apresentou conteúdo de açúcares totais aproximados à farinha de aveia que possui $67,89 \%$ de carboidratos em sua composição, segundo dados desses autores.

\section{4 - CONCLUSÕES}

- As características agronômicas apresentadas pela cultura do sorgo indicam a sua potencialidade como grande produtor de biomassa, sementes e folhas, elevada capacidade de rendimento de colmos, alta percentagem de extração de caldo, o qual possui alto conteúdo de sólidos solúveis totais, similar ao da cana-de-açúcar.

- $\quad$ Rapaduras em que o caldo de sorgo entrou na proporção de 10 e $20 \%$ obtiveram aceitação geral superior a rapaduras produzidas a partir de $100 \%$ caldo de cana-de-açúcar.

- Rapaduras em que o caldo de sorgo entrou na proporção de $30 \%$ apresentaram aceitação geral similar a rapaduras produzidas a partir de $100 \%$ caldo de cana-de-açúcar. Produtos formulados com proporções de caldo do colmo de sorgo superiores a 30\% não apresentaram cristalização e consistência satisfatórias para obtenção de rapadura.

- Nas combinações caldo de sorgo x caldo de cana, à medida que a proporção de sorgo aumentava, os teores de cinzas e umidade nas rapaduras aumentavam e os valores de acidez tornavam-se menores.

- O melaço produzido a partir de $100 \%$ caldo de sorgo produziu melaço com elevados teores de açúcares totais, umidade e cinzas.

- A farinha obtida de três tipos de grãos de sorgo apresentaram valores significativos em relação ao teor de cinzas e açúcares totais com $\mathrm{pH}$ próximo da neutralidade.

- O caldo do colmo do sorgo pode ser utilizado como matéria-prima complementar para a produção de rapadura e os grãos podem ser utilizados para a preparação de farinha, caracterizando o sorgo sacarino como uma alternativa válida para utilização como alimento, constituindo-se também em uma outra possibilidade na produção agropecuária do Nordeste.

\section{5 - REFERÊNCIAS BIBLIOGRÁFICAS}

[1] ANVISA. Agência Nacional de Vigilância Sanitária. Resolução CNNPA no. 12, 24/07/1978. www.anvisa.gov.br/legis/resol, acessado em 11/05/2005.

[2] BRASIL, Portaria n. 354, de 18 de julho de 1996. Diário Oficial da União, Brasília, 22 de julho de 1996.

[3] BYE, P.; MEUNIER, A.; MUCHNIK, J. As inovações açucareiras: permanência e diversidade de paradigmas. Cadernos de Ciência e Tecnologia, v. 10, n.1/3, p. 3552, 1993.

[4] CAMACHO, R.; MALAVOLTA, E.; GUEIREIRO-ALVES, J.; CAMACHO, T. Vegetative growth of grain sorghum in response to phosphorus nutrition. Scientia Agricola, v.59, n. 4. p. 771-776, 2002.

[5] CARVALHO, N. M.; NAKAGAWA, J. Sementes, Ciência, Tecnologia e Produção, $2^{a}$ edição. Campinas, Fundação Cargill, 1983. p. 7, 55 e 61.

[6] CHIELLE, Z. Efeito da irrigação duas cultivares de sorgo sacarino e quatro densidades de plantio em solos de várzea. In: Reunião Técnica Anual do Sorgo, XIII , 1984. Anais..., Pelotas RS, 1984.

[7] GUTKOSKI, L. C.; NETO, R. J. Procedimento para teste laboratorial de panificação pão tipo forma. Ciência 
Rural, v. 32, n. 5, p. 873-879, 2002

[8] INSTITUTO ADOLFO LUTZ Normas analiticas do Instituto Adolfo Lutz. $3^{\text {a }}$ edição, São Paulo: Instituto Adolfo Lutz, 1985. 533p.

[9] LIPINSKI, E.S.; KRESOVICH, S. Sugar crops as a solar energy converters. Experimentia, v.38, p.13-17, 1992.

[10] LIRA, M. A. Cultivo do Sorgo [Sorghum bicolor ( L. ) Moench]. Recife, Empresa Pernambucana de Pesquisa Agropecuária, 1983.4p.

[11] MARCHEZAN, E. Resultados do Ensaio Nacional do Sorgo em Santa Maria, RS. Reunião Técnica Anual do Sorgo, XVI, 1987. Anais... Pelotas-RS, 1987.

[12] NEUMANN, M.; RESTlE, J.; ALVES FILHO, D.C.; BRONDANI, I.L.; MENEZES, L.F.G. Resposta econômica da terminação de novilhos em confinamento, alimentados com silagem de diferentes híbridos de sorgo (Sorghum bicolor, L. Moench). Ciência Rural, v. 32, n.5., p. 123-133, 2002.
[13] OliveirA, F. M. Consumo Humano do Sorgo na Propriedade Agrícola. Sorgo, Uma Opção Agrícola Informe Agropecuário, n. 144, p. 11 13, 1986.

[14] RASPER, V. F. Quality evaluation of cereal and cereal products. In: LORENZ, K. J.; KULP, K. (ed). Handbook of cereal science and technology. New York: Marcel Dekker, 1991, p. 592-638.

[15] TEIXEIRA, C.G.; JARDINE, J. G.; BEISMAN, D.A. Utilização do sorgo sacarino como matéria-prima complementar à cana-de-açúcar para obtenção de etanol em microdestilaria. Ciênc. Tecnol. Aliment., v. 17, n.3, p. 221-229, 1997.

[16] TREDUS, A. S., ORMENES, R. de C. S. C.; SPERANZA, S. M.; CHANG, Y. K.; BUSTOS, F. M. Estudo da adição de vital glúten à farinha de arroz, farinha de aveia, amido de trigo na qualidade de pães. Ciênc. Tecnol. Aliment., v. 21 , n. 1, p. 20-25, 2001. 